infrared detectors for imaging applications, IEEE JSTQE 11 (2005), $117-120$.

4. F.J. González, C.S. Ashley, P.G. Clem, and G.D. Boreman, Antennacoupled microbolometer arrays with aerogel thermal isolation, Infrared Phys Tech 45 (2004), 47-51.

5. F.J. González, M. Abdel-Rahman, and G.D. Boreman, Antenna-coupled VOx thin-film microbolometer array, Microwave Opt Technol Lett 38 (2003), 235-237.

6. D.P. Neikirk and D.B. Rutledge, Air-bridge microbolometer for far infrared detection, Appl Phys Lett 44 (1984), 153-155.

7. M. Elwenspoek, and R. Wiegerink, Mechanical Microsensors, Springer-Verlag, 2001.

8. R.L. Edwards, G. Coles, and W.N. Sharpe, Jr., Comparison of tensile and bulge tests for thin-film silicon nitride, Experimen Mechan 44 (2004), 49-54.

9. H. Huang, K. Winchester, Y. Liu, X.Z. Hu, C.A. Musca, J.M. Dell, and L. Faraone, Determination of mechanical properties of PECVD silicon nitride thin films for tunable MEMS Fabry-Pèrot optical filters, J Micromech Microengg 15 (2005), 608-614.

10. M.A. Gritz, I. Puscasu, D. Spencer, et al., Fabrication of an infrared antenna-coupled microbolometer linear array using chrome as a mask, JVST B 21 (2003), 2608-2611.

(C) 2005 Wiley Periodicals, Inc.

\section{EXTENSION OF THE ANALYTICAL MODELLING OF RESISTIVE LOADED THIN-WIRE ANTENNAS TO GAUSSIAN DERIVATIVES EXCITATIONS}

\author{
F. Sagnard ${ }^{1,2}$ and C. Vignat $^{2}$ \\ ${ }^{1}$ IETR, UMR 6164, INSA \\ 20, avenue des Buttes de Coësmes \\ 35043 Rennes, France \\ 2 IGM, Université de Marne-La-Vallée \\ 77454 Marne-La-Vallée cedex 2, France
}

\section{Received 10 June 2005}

\begin{abstract}
The transient electric field radiated from a thin-wire dipole antenna resistively loaded and excited by a voltage signal in the shape of the Gaussian derivatives is analyzed. The modelling presented in this paper is formulated in the time domain using an inverse Fourier transform of the analytical expressions obtained by Wu and King in the frequency domain. Such a modelling appears as an extension of previous developments, as it allows us to consider any Gaussian derivatives as excitation signals as well as the presence of a nonnegligible impedance in the feeding circuit. Parameter studies are presented to analyze the waveforms and the energy radiated in the far-field zone. () 2005 Wiley Periodicals, Inc. Microwave Opt Technol Lett 47: 548-553, 2005; Published online in Wiley InterScience (www.interscience.wiley. com). DOI 10.1002/mop.21227
\end{abstract}

Key words: broadband antenna; Gaussian excitation; thin-wire antenna; transient response; electromagnetic radiation

\section{INTRODUCTION}

Wireless data transmission at high rate, short range, and low cost appears to be an alternative for numerous applications dealing with communications, networking, radar, imaging, and positioning systems. Ultra-wideband (UWB) technology provides significant potential, as it relies on the radiation and propagation of extremely large baseband pulses characterized by a spectral occupancy in excess of $500 \mathrm{MHz}$ and a fractional bandwidth of more than 20\% [1]. As this technology aims to maximize the utilization of underused spectrum segments with- out licence, and to minimize interference with other communication systems, the maximum allowable power spectral density (PSD) should be specified. Thus, in February 2002, the FCC defined a spectral mask, and the ETSI is currently working on a similar mask which appears to be more restrictive. For the design and evaluation of an UWB communication link, critical points are evaluation of the energy loss and the waveform distortions experienced by the transmitted signal which has propagated through a multipath channel [2].

In fact, the transmitting and receiving antennas appear to influence the overall performances of UWB systems significantly. In particular, the UWB transmitter and receiver should ideally have flat responses and linear phase variations versus frequency over the frequency band of interest. Deviations from these constraints will cause distortion in the initial signal. In this paper, we focus our studies on the transient waveforms radiated in the transmission configuration by thin-wire antennas when they are excited by a short transient signal generated by a voltage source. The voltage signal is assumed to have the shape of the Gaussian function or one of its successive derivatives. To correlate and analyze the waveforms radiated with the physical phenomena involved, we consider the basic dipole antenna because it can be described using analytical relations. Moreover, such an antenna allows us to consider an assembly of thin-wire elements in order to present approximated models of antennas such as the V-dipole, the bow-tie antenna, and the TEM hornlike antenna. The dipoles studied include a passive load to prevent reflection at the top end in order to satisfy broadband characteristics. Such a behaviour can be obtained by different means; the loading technique proposed by $\mathrm{Wu}$ and King in 1965, which considers a distributed resistive profile along the antenna, has been found to be very efficient [3]. The time-domain modelling proposed in this paper appears as an extension of previous studies, as it allows us to consider all voltage excitations which have the shape of the Gaussian function and its successive derivatives. Moreover, an equivalent impedance associated with the input circuit has been included in the modelling. The electric-field waveforms radiated are calculated and analyzed in the far-field zone. The parametric studies presented demonstrate the influence, on the waveforms and the energy radiated, of the duration and the shape of the incident pulse, the source impedance, and the antenna geometry. Such studies form an aid to improve the physical phenomena involved in the transient radiation of more complex ultra-wideband antennas.

\section{TRANSIENT RESPONSE OF A DIPOLE ANTENNA}

\subsection{General Principles}

The dipole antenna is made of two finite-length thin cylinders (or arms) of length $L$; it is assumed to be initially centred and aligned with the $O z$ axis so that the feed point is positioned at $z=0$. It is included in an electric circuit, as shown in Figure 1. A voltage generator $V_{g}$ is supposed to deliver an excitation signal in the equivalent circuit made of three impedances: the source impedance $\left(Z_{g}\right)$, the impedance of the transmission line linking the generator to the antenna feed-point $\left(Z_{l}\right)$, and the input antenna impedance at the feed-point $Z_{A}$ [4]. In general, all these impedances depend on frequency. In the circuit, the presence of nonuniform impedances and impedance mismatches over the frequency bandwidth of the excitation signal causes signal distortions. In the frequency domain, the relation between the input voltage and the resulting current is given by 


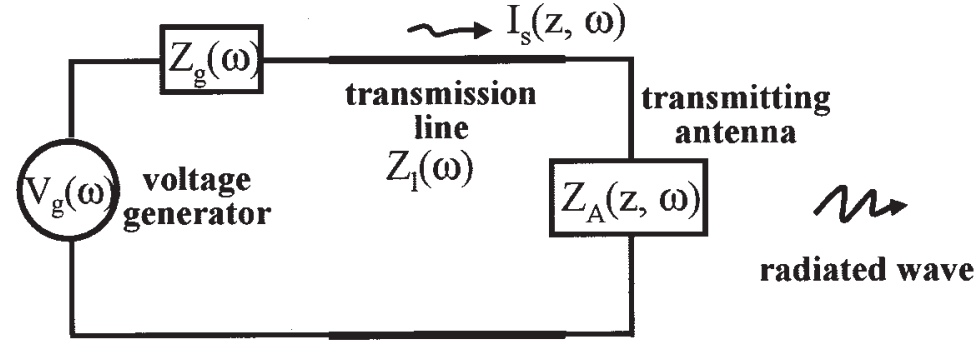

(a)

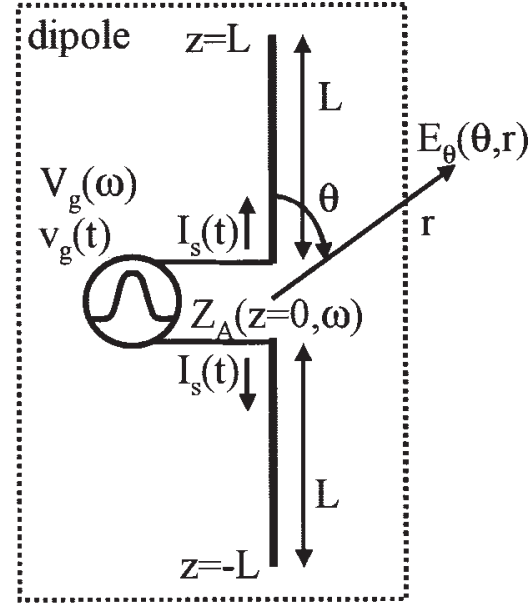

(b)

Figure 1 (a) General scheme of a transmitting antenna and its feeding circuit and (b) the geometry of a center-feed dipole antenna

$$
I_{s}(\omega)=\frac{V_{g}(\omega)}{Z_{t}(\omega)}
$$

where $Z_{t}(\omega)=Z_{g}(\omega)+Z_{l}(\omega)+Z_{A}(\omega)$

For simplicity, we will assume hereinafter that $Z_{g}$ and $Z_{l}$ are independent of the frequency.

In the time domain, we consider that the excitation voltage has the shape of an $n^{\text {th }}$ differentiated Gaussian pulse $g(t)$, given by

$$
V_{g}(t)=g^{(n)}(t)=\frac{d^{n}}{d t^{n}}\left(A_{0} e^{-(t / \tau)^{2}}\right)
$$

where $\tau$ represents the characteristic time of the pulse and $A_{0}$ is the voltage amplitude. The pulse duration $w$ of each signal is defined as the time interval on either side of the maximum amplitude where $99 \%$ of the total energy of the pulse is contained. For example, for $n=0, w=4 \tau$; for $n=1, w=4.6 \tau$; and for $n=$ $2, w=5.05 \tau$.

Such a signal can be expressed using the Hermite polynomial of degree $n$ as follows:

$$
g^{(n)}(t)=A_{0}\left(-\frac{1}{\tau}\right)^{n} H_{n}\left(\frac{t}{\tau}\right) e^{-(t / \tau)^{2}}
$$

\subsection{The Resistive Loaded Dipole}

To prevent reflection at both ends $z=L$ and $z=-L$ of the antenna, a distributed passive loading along each arm is used that progressively reduces the current amplitude to an insignificant value at both ends. A possible method to obtain such a condition is to use a nonuniform resistive load, as a reactive loading (being frequency sensitive) would cause distortion to the current wave; for physical considerations, a uniform resistive loading is incapable of eliminating current reflection at the ends, although it may appreciably reduce the effects of such reflections. The solution for a nonreflecting dipole was first proposed by Wu and King (WK) in 1965 [3], and since then it has been extended to several applications $[5,6]$. The modelling has been formulated in the frequency domain and assumes that an internal impedance is variable along the dipole and made of resistive and capacitive components. In the case of a short dipole, the distributed capacitance appears much smaller than the resistive component. Thus, only a distributed resistive loading per unit length $z$ along the dipole has been considered [3]:

$$
Z_{A}(z, \omega)=r(0)\left(\frac{L}{L-|z|}\right) \Omega
$$

where $r(0)$ is the value of the distributed resistance at the feed point $z=0$ of the dipole and $\psi$ is a parameter evaluated by WK as follows:

$$
\begin{aligned}
r(0)=\frac{\eta_{0} \psi}{2 \pi}=60 \psi \Omega, \psi & \approx 2\left[\sinh ^{-1}\left(\frac{L}{a}\right)-C(2 k a, 2 k L)\right. \\
& -j S(2 k a, 2 k L)]+\frac{j}{k L}\left(1-e^{-j 2 k L}\right),
\end{aligned}
$$

where $k=\omega / c$ is the wave number in the vacuum, $\eta_{0}$ is the impedance of free space $(120 \pi)$, and $C(2 k a, 2 k L)$ and $S(2 k a$, $2 k L)$ are the generalized sine and cosine integrals, respectively, given by

$$
C(2 k a, 2 k L)=\int_{0}^{2 k L} \frac{1-\cos W}{W} d u ; S(2 k a, 2 k L)=\int_{0}^{2 k L} \frac{\sin W}{W} d u
$$

and

$$
W=\left(u^{2}+(2 k a)^{2}\right)^{1 / 2}
$$

The current along the dipole is first expressed in the frequency domain and, contrary to previous studies, we assume a nonzero impedance value for $Z_{g}\left(Z_{L}=0\right)$ :

$$
I_{s}(z, \omega)=\frac{V_{g}(\omega)}{Z_{g}+Z_{A}(z, \omega)},
$$

which gives 


$$
\begin{aligned}
I_{s}(z, \omega) & =\frac{2 \pi}{\eta_{0} \psi}\left(\frac{\omega V_{g}(\omega)}{\omega\left(1+2 \pi Z_{g} /\left(\eta_{0} \psi\right)\right)-j / \tau_{a}}\right)\left(1-\frac{|z|}{L}\right) e^{-j k|z|} \\
& =\frac{2 \pi}{\eta_{0} \psi C_{g}}\left(\frac{\omega V_{g}(\omega)}{\omega-j / \tau_{t}}\right)\left(1-\frac{|z|}{L}\right) e^{-j k|z|}
\end{aligned}
$$

where $\omega=2 \pi f$ is the angular frequency, $\tau_{a}=L / c, C_{g}=1+$ $2 \pi Z_{g} /\left(\eta_{o} \psi\right)$, and $\tau_{t}=C_{g} \tau_{a}$. The time-dependent current along the dipole can be written as

$$
\begin{aligned}
I_{s}(z, t)=F T^{-1}\left(I_{s}(z, \omega)\right)= & \frac{2 \pi}{\eta_{0} \psi C_{g}}\left(1-\frac{|z|}{L}\right)\left[V_{g}\left(t_{z}\right)\right. \\
& \left.-\frac{1}{\tau_{t}} \int_{0}^{\infty} V_{g}\left(t_{z}-t^{\prime}\right) e^{-t^{\prime} / \tau_{t}} d t^{\prime}\right],
\end{aligned}
$$

where $F T^{-1}$ denotes the inverse Fourier transform and $t_{z}=t-$ $|z| / c$.

Then, in the frequency domain, the radiated electric field $E_{\theta}(r$, $\theta, \omega)$ in the far-field zone can be expressed as a function of the spherical coordinates $(r, \theta)[3,6]$ :

$$
E_{\theta}(r, \theta, \omega)=j \frac{\eta_{0} L C(\omega) e^{-j k r}}{2 \pi r} F(k L, \theta)
$$

where

$$
C(\omega)=-\frac{2 \pi \varepsilon_{0}}{\psi} \frac{\omega V_{g}(\omega)}{\left(j-k L\left(1+2 \pi Z_{g}\right)\right)}=\frac{2 \pi \varepsilon_{0}}{\psi \tau_{t}} \frac{\omega V_{g}(\omega)}{\left(\omega-j / \tau_{t}\right)}
$$

and

$$
\begin{aligned}
& F(k h, \theta)=\frac{1}{k L \sin ^{3} \theta}\left[-j k L \sin ^{2} \theta+\left(1+\cos ^{2} \theta\right)\right. \\
& \left.-\frac{1}{2}(1+\cos \theta)^{2} e^{-j k L(1-\cos \theta)}-\frac{1}{2}(1-\cos \theta)^{2} e^{-j k L(1+\cos \theta)}\right] .
\end{aligned}
$$

Thus, Eq. (10) of the field $E_{\theta}(r, \theta, \omega)$ can be rewritten as follows:

$$
E_{\theta}(r, \theta, \omega)=\frac{\tau_{a} e^{-j k r}}{\psi \tau_{t} r} \frac{j \omega V_{g}(\omega)}{\left(\omega-j / \tau_{t}\right)} F(k L, \theta)
$$

Then, the transient radiated electric field in the far-field zone is expressed using the inverse Fourier transform $T F^{-1}\left(E_{\theta}(r, \theta, \omega)\right)$, given by

$$
=\frac{1}{r \psi \sin ^{3} \theta} \frac{\tau_{a}}{\tau_{t}}\left[\begin{array}{c}
\sin ^{2} \theta\left(V_{g}\left(t^{*}\right)-\frac{1}{\tau_{t}} \int_{0}^{\infty} V_{g}\left(t^{*}-t^{\prime}\right) e^{-t^{\prime} / \tau_{t}} d t^{\prime}\right) \\
-\frac{\left(1+\cos ^{2} \theta\right)}{\tau_{t}} \int_{0}^{\infty} V_{g}\left(t^{*}-t^{\prime}\right) e^{-t^{\prime} / \tau_{t}} d t^{\prime} \\
+\frac{(1+\cos \theta)^{2}}{2 \tau_{t}} \int_{0}^{\infty} V_{g}\left(t_{1}-t^{\prime}\right) e^{-t^{\prime} / \tau_{t}} d t^{\prime} \\
+\frac{(1-\cos \theta)^{2}}{2 \tau_{t}} \int_{0}^{\infty} V_{g}\left(t_{2}-t^{\prime}\right) e^{-t^{\prime} / \tau_{t}} d t^{\prime}
\end{array}\right],
$$

where

$$
t^{*}=t-\frac{r}{c} ; t_{1}=t^{*}-\tau_{a}(1-\cos \theta) ; t_{2}=t^{*}-\tau_{a}(1+\cos \theta) .
$$

We remark that if $Z_{g}=0$, we have $\tau_{t}=\tau_{a}$.

The radiated electric field in Eq. (14) includes four terms associated to each line of the expression. The first and the second terms correspond to a radiation issued from the feed point $z=0$. The integral terms express the convolution of the exciting voltage $V_{g}(t)$ and an exponentially decaying factor, thus representing a distorted replica of the exciting input due to the resistive loading. The third and fourth terms are interpreted as radiated signals issued from the top and bottom ends, respectively. The associated path length are expressed by the delays $t_{1}$ and $t_{2}$. So, the four terms decomposition in Eq. (14) can be used to model more complex geometry, such as a $\mathrm{V}$-dipole, by rotating both arms properly.

As we assume that the excitation voltage is described by Eq. (2), the general integral $\int_{0}^{\infty} V_{g}(y-x) e^{-x / \tau_{t}} d t^{\prime}$ met in relation (14) can be computed using iterated integration by parts and relation (3) as follows:

$\int_{0}^{\infty} \frac{d^{n}}{d t^{n}}\left(e^{-(x-y / \tau)^{2}}\right)\left(e^{-\left(x / \tau_{t}\right)}\right) d x$

$$
\begin{aligned}
=\sum_{p=0}^{n-1}(-1)^{p}\left[\frac{(-1)^{n-p}}{\tau^{n-p-1} \tau_{t}^{p}} H_{n-p-1}\left(\frac{-y}{\tau}\right) e^{-(y / \tau)^{2}}\right] \\
+\left(\frac{1}{\tau_{t}}\right)^{n} \int_{0}^{\infty} e^{-(x-y / \tau)^{2}} e^{-\left(x / \tau_{t}\right)} d x .
\end{aligned}
$$

The right-hand side integral is written as

$$
\int_{0}^{\infty} e^{-(y-x / \tau)^{2}} e^{-\left(x / \tau_{t}\right)} d x=\tau \frac{\sqrt{\pi}}{2} \operatorname{erfc}\left(-\frac{y}{\tau}+\frac{\tau}{2 \tau_{t}}\right) e^{\left(-\left(y / \tau_{t}\right)+\left(\tau^{2} / 4 \tau_{t}^{2}\right)\right)},
$$

where $\operatorname{erfc}(y)=2 / \sqrt{\pi} \int_{y}^{\infty} e^{-t^{2}} d t$ is the error function.

\section{ANALYSIS OF SIMULATION RESULTS}

\subsection{Initial Conditions}

The basic dipole considered is characterized by its arm length $L$ of $10 \mathrm{~cm}$ and a length-to-radius ratio of $10^{-4}$. The traveling time associated with each of the two arm lengths is $\tau_{a}=L / c=$ $33.3 \mathrm{~ns}$. The far-field component $E_{\theta}(r, \theta, \omega)$ of the electric field radiated is analyzed at the distance $r=1 \mathrm{~m}$. The voltage amplitude $A_{0}$ has been fixed to $1 \mathrm{~V}$ and several generator impedance values for have been considered $Z_{g}=0 \Omega, 100 \Omega$, $1000 \Omega$. Several values have been considered for the characteristic time $\tau$ associated with the set of Gaussian excitations; these values have been set in order to fit $n=1,2$, or 4 pulse durations (defined as the time in which $99 \%$ of the total energy of the pulse is contained) in an arm length $L$. So, the case of a Gaussian pulse $\tau$ will take the values $\tau_{1}=83.3 \mathrm{ps}, \tau_{2}=41.7$ ps, $\tau_{3}=20.8 \mathrm{ps}$, respectively, and in the case of the first derivative of the Gaussian function, the associated values will be $\tau_{1}^{\prime}=72.5 \mathrm{ps}, \tau_{2}^{\prime}=36.2 \mathrm{ps}$, and $\tau_{3}^{\prime}=18.1 \mathrm{ps}$. As the parameter $\psi$ varies as a function of the frequency, we have 


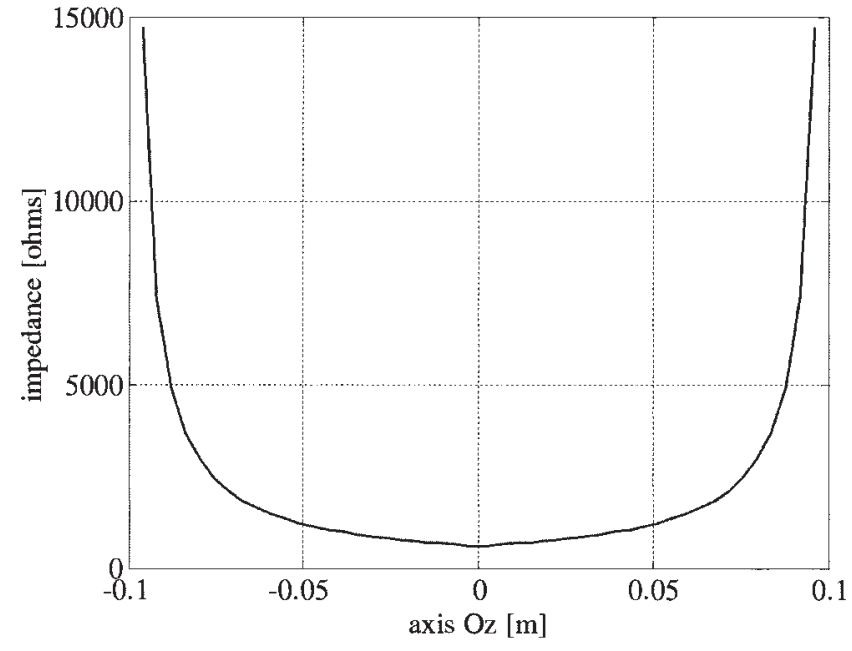

Figure 2 Impedance distribution along the dipole antenna $(-L, L)$ with arm length $L=10 \mathrm{~cm}(\psi=10)$

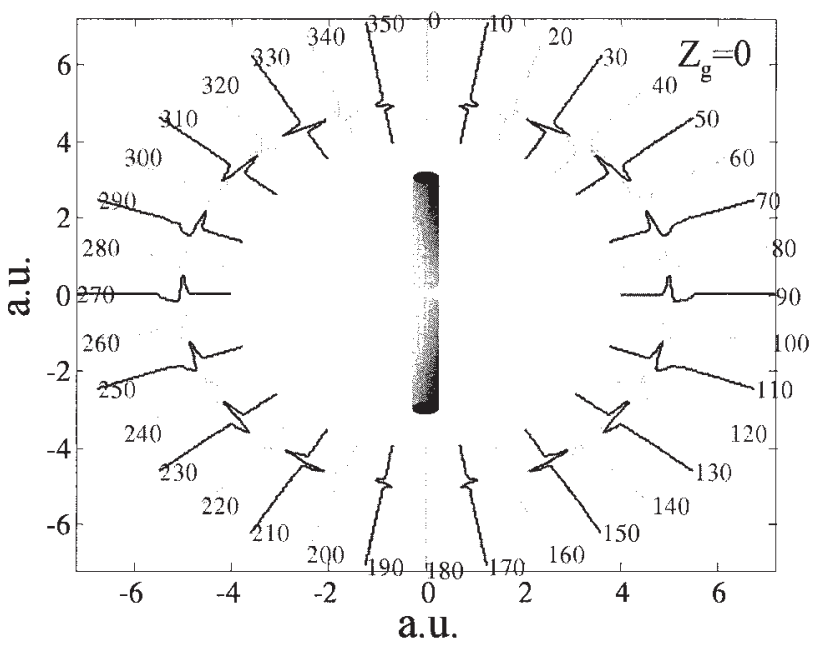

(a)

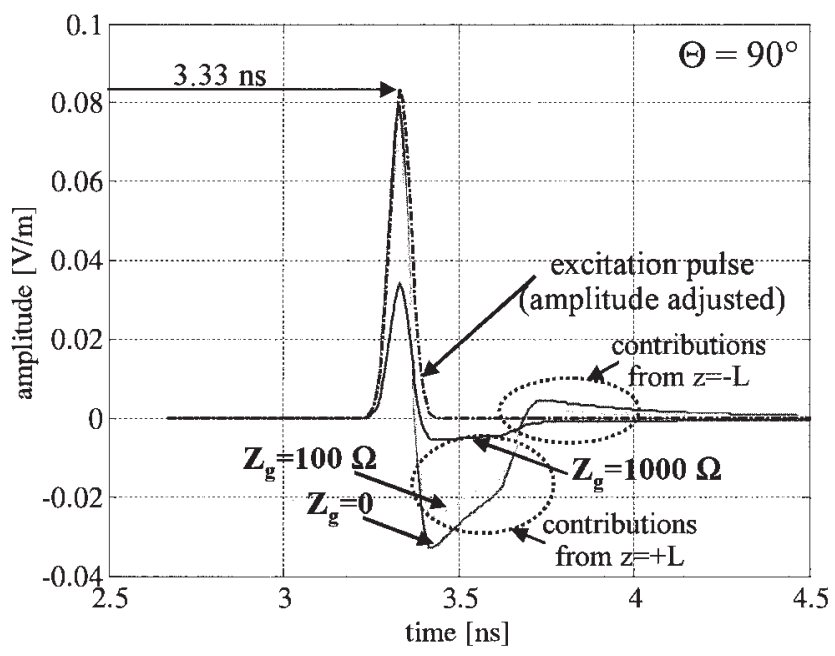

(b)

Figure 3 The spatio-temporal electric field radiated by a straight dipole ( $L=10 \mathrm{~cm}, n=2$ ) excited by a Gaussian pulse (a) in the plane of the antenna $\left(Z_{g}=0\right)$ and (b) in the direction $\theta=90^{\circ}$ considering the generator impedances $Z_{g}=0 \Omega, 100 \Omega$ and $1000 \Omega$

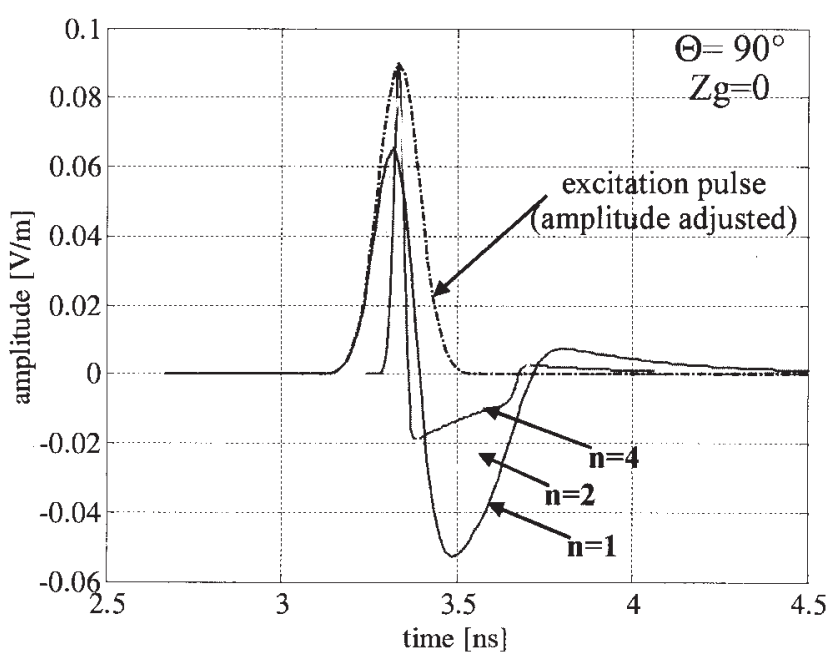

(a)

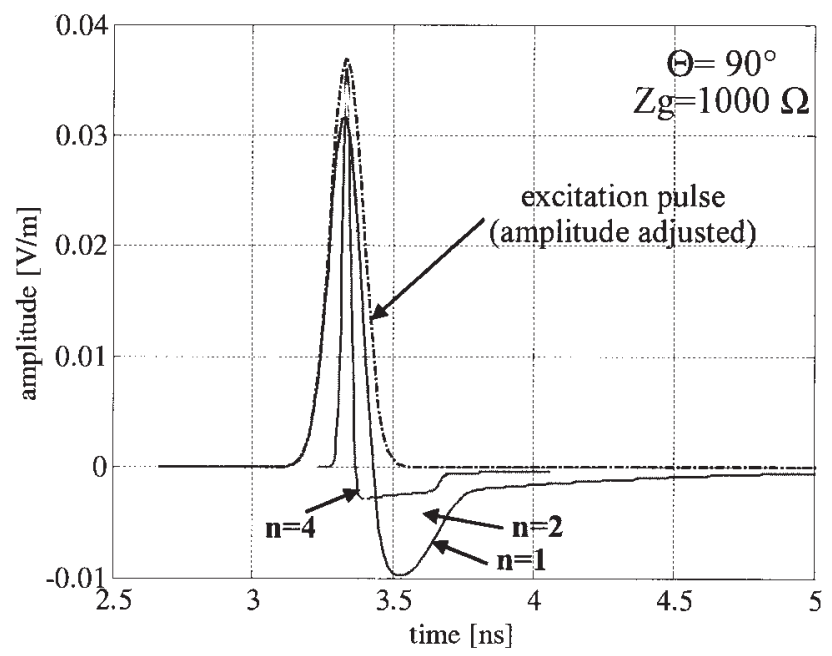

(b)

Figure 4 The electric field radiated by a straight dipole $(L=10 \mathrm{~cm})$ excited by a Gaussian pulse for several characteristic times in the direction $\theta=90^{\circ}$ considering (a) $Z_{g}=0 \Omega$ and (b) $Z_{g}=1000 \Omega$

replaced it by a mean value estimated to 10 , corresponding to the initial simulation conditions defined previously $[5,6]$. Thus, the impedance of the antenna at the feed point is $r(0)=600 \Omega$; the impedance distribution along the dipole antenna $(-L, L)$ shown in Figure 2, highlights that its value increases rapidly near the ends, and tends to infinity at the ends.

\subsection{Parametric Studies in the Case of a Straight Dipole}

In this section, we report several results of parametric studies obtained using our user-friendly interface.

In the case of a Gaussian excitation voltage, the influence of the characteristic time $\tau$ and the generator impedance $Z_{g}$ on the spatio-temporal waveforms radiated by the dipole antenna has been studied. Considering the characteristic time $\tau_{2}=41.7 \mathrm{ps}$ $(n=2)$ and the generator impedance $Z_{g}=0$, the electric radiated field shown in Figure 3(a) shows that a positive pulse issued from the feed point $z=0$ is firstly radiated and then followed by a negative pulse issued from the radiations at both ends $z=-L$ and $L$, the delays of which depend on the observation angle. We remark that the amplitude of the positive pulse 


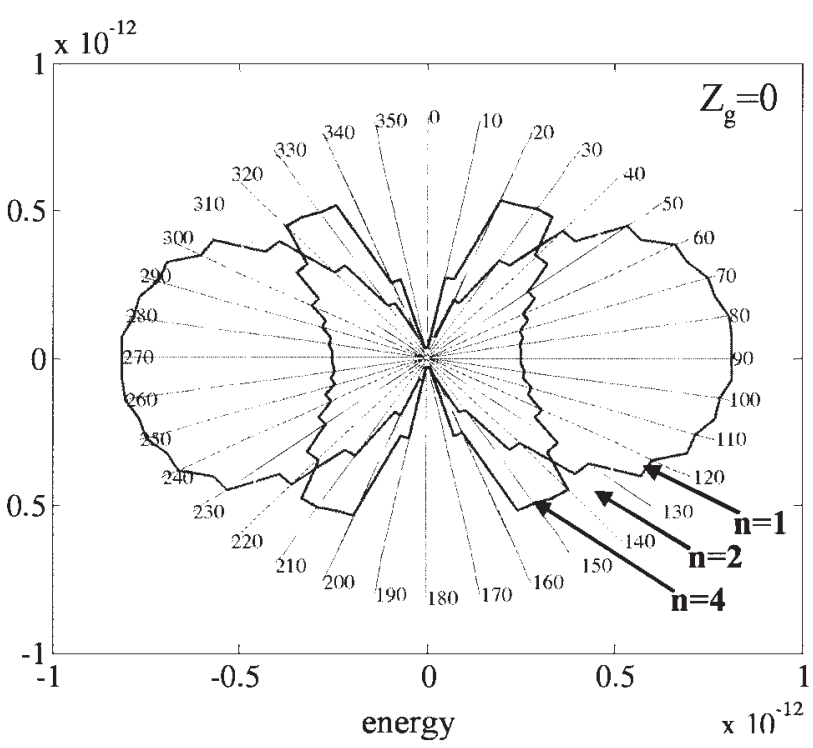

(a)

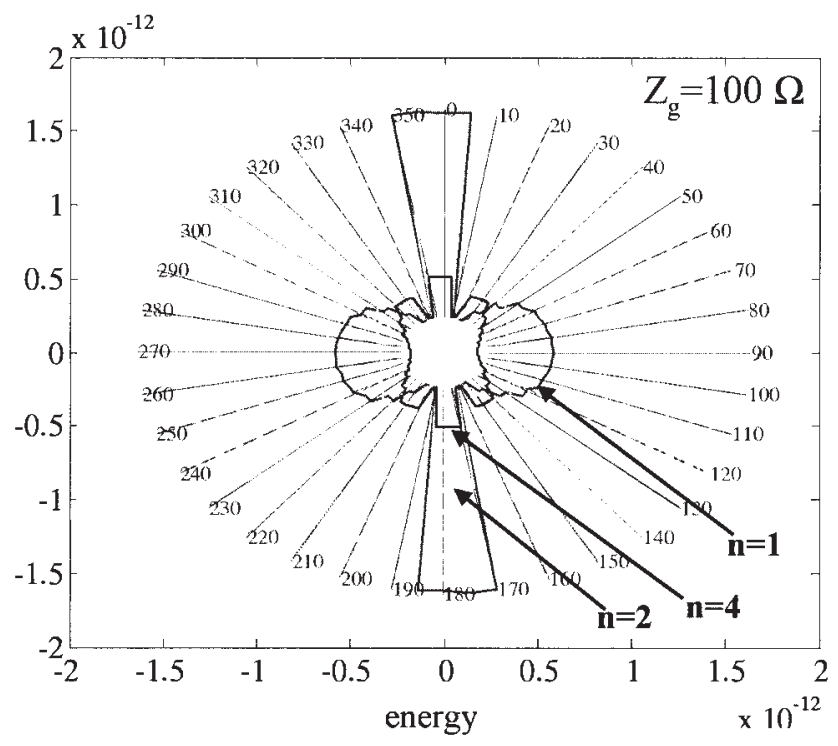

(b)

Figure 5 Electric energy $\left(E^{2}\right)$ radiated in the plane of the dipole for several characteristic times considering in the case of a Gaussian excitation: (a) $Z_{g}=0 \Omega$; (b) $Z_{g}=100 \Omega$

depends on the observation angle, and that distortion appears between $\theta=30^{\circ}$ and $40^{\circ}$ as the negative pulse moves closer to the positive one. Also, we notice that the relative amplitude of the negative pulse compared to the positive one appears to be weaker for the direction $\theta=90^{\circ}$; thus, the attenuation caused by the resistive load is the most effective. In Figure 3(b), the contributions issued from the ends $z=-L$ and $L$ are indicated; the positive pulse issued from the feed point seems to reproduce well the incident voltage signal. In the direction $\theta=90^{\circ}$, the study of the influence of the generator impedance $Z_{g}$ highlights that increasing values cause attenuation and distortion of the radiated signals. Also, the undershoot and its following overshoot clearly visible for $Z_{g}=0$ appear significantly smoothed for $Z_{g}=1000 \Omega$. This observation is confirmed in Figures 4(a) and 4(b), where the influence of the characteristic time has been studied for both values of $Z_{g}$. Moreover, we observe that a longer negative tail is visible for $Z_{g}=1000 \Omega$ (with a longer decay time $\tau_{t}$ for this impedance). As a short excitation pulse (with a duration shorter than the traveling time associated with length $L$, as for $n=4$ or $n=2$ ) is responsible for the differentiating effect induced by the dipole, an excitation with a duration close or greater than the time necessary for a wave to travel the length $L$ induces an integration effect [see Figs. 4(a) and 4(b)]. The shape of the radiated energy $P(\theta)=\int E_{2 \theta}^{2}(\theta, t) d t$ in the plane of the dipole has been studied as a function of the impedance $Z_{g}$, as shown in Figures 5(a) and 5(b); note that when the value increases from $0 \Omega$ to $100 \Omega$, the maximum shifts towards the direction $\theta=0^{\circ}$. This phenomenon can be explained by a greater radiation attenuation induced at both ends $z=-L$ and $L$ and caused by the presence of an additional resistance in the antenna circuit. So, the positive pulse radiated by the feed point appears more involved in the radiation. Moreover, Figure 5(a) shows that if $Z_{g}=0$, the pulse duration associated with $n=1\left(\tau_{1}=83.3 \mathrm{ps}\right)$ produces a nearly omnidirectional radiation pattern; however, if the pulse duration is shorter, the focusing appears more definite in the directions $\theta$ ranging from $25^{\circ}$ to $45^{\circ}$. These energy patterns have been compared with the energy pattern associated with an infinitively conductive dipole [7]. For comparison with the loaded dipole, such a dipole is supposed to have an impedance of $600 \Omega$ at the feed point; also, we have assumed a double-passing excitation mode, which implies that radiation occurs once at the feed point and the ends. Thus, the pattern shown in Figure 6 can be compared with the pattern of Figure 5(a). The comparison highlights that a smoothing effect is observed with the loaded dipole and local maxima are only remarked in the case of the infinitively conductive dipole. Of course, the radiation efficiency is lower in the case of the loaded dipole; when $Z_{g}=0$, $\mathrm{Wu}$ and King have evaluated the efficiency to $50 \%$.

In the case of a first differentiated Gaussian excitation voltage, samples of radiated waveforms with $Z_{g}=100 \Omega$ and $\tau_{2}^{\prime}=36.2 \mathrm{ps}$ $(n=2)$ have been plotted, as shown in Figure 7(b). An additional

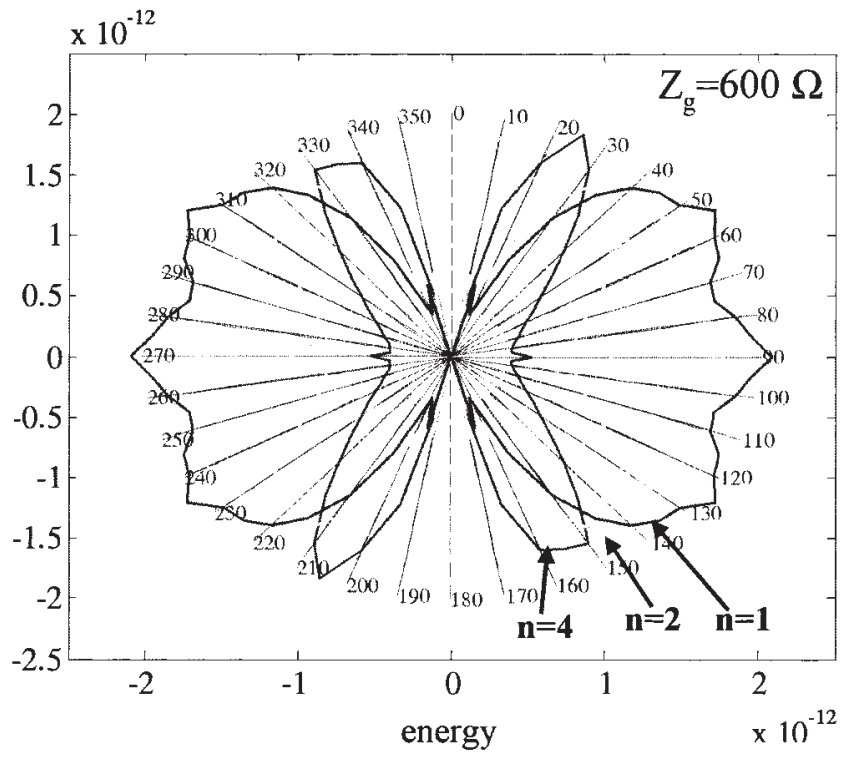

Figure 6 Electric energy $\left(E^{2}\right)$ radiated by an infinitively conductive dipole for several characteristic times $\tau$ in the case of a Gaussian excitation $\left(L=10 \mathrm{~cm}, Z_{g}=600 \Omega\right)$ 


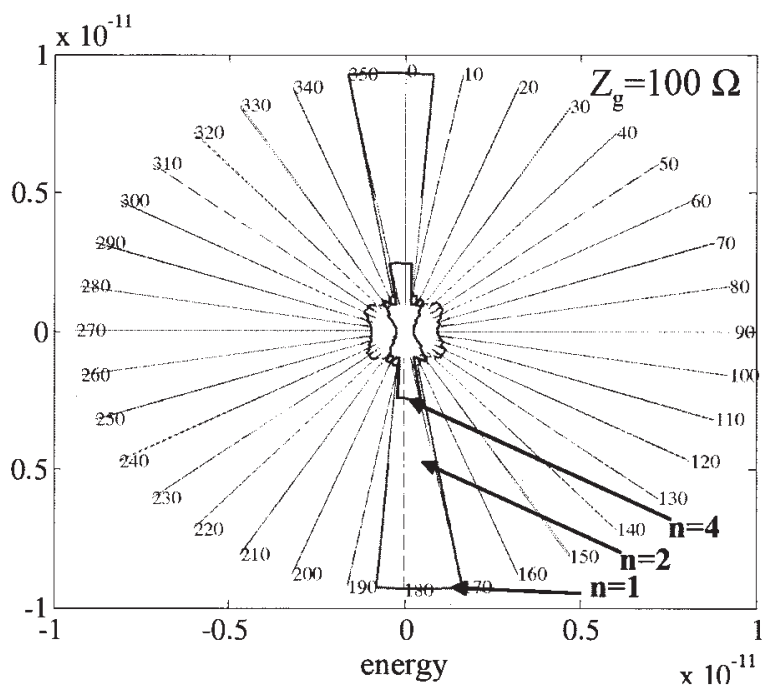

(a)

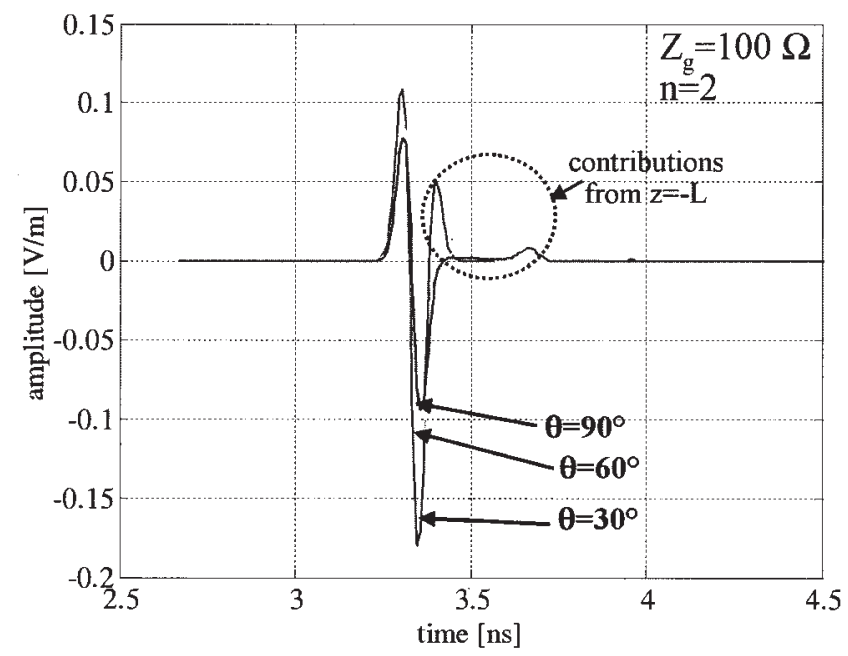

(b)

Figure 7 (a) Electric energy $\left(E^{2}\right)$ radiated in the case of the first derivative Gaussian excitation for several characteristic times $\left(Z_{g}=100 \Omega\right)$ and (b) the waveforms as a function of the observation angle $\theta$

overshoot can be visualized which corresponds to the field radiated by the end positioned at $z=+L$. The contribution of the end at $z=-L$ is included in the negative pulse. Thus, in that case, the radiated waveforms appear more difficult to analyze because of the presence of two signed components in the input signal. The energy pattern shows a similar shape with the pattern associated with the Gaussian input signal presented in Figure 5(b).

\section{CONCLUSION}

The analytical modelling of a resistive loaded dipole initially proposed by $\mathrm{Wu}$ and King in the frequency domain has been revisited in order to consider an excitation voltage in the set of differentiated Gaussian functions. Also, the presence of an equivalent impedance associated with the input circuit has been considered. In the modelling presented, the electric field radiated by a dipole leads to a spatio-temporal response that is formulated analytically. The formulation, based on the use of the Hermite polynomials, contains four components associated with two signals issued from the feed point and two signals coming from both arm ends. Some components from the feed point appear as a distorted replica of the initial pulse by the presence of an exponential decaying function. The pulses radiated from the ends appear also a distorted replica of the initial signal, but at a delayed time.

The user-friendly software associated with the modelling allows us to perform several parameter studies and to highlight the physical phenomena involved by a resistive loaded dipole. The results presented highlight the main observations: a generator impedance induces an additional attenuation and a delayed tail in the radiated waveforms; also, the resistive load is responsible for an additional distortion which overlaps the distortion induced by the differences of wave paths reaching the observation point. Moreover, it must be remarked that the ratio $R_{w}$ of the pulse duration to the travel time associated with the propagation along each arm determines the antenna behaviour; as a short pulse has a differential effect $\left(R_{w}<1\right)$, a longer pulse $\left(R_{w} \geq 1\right)$ has an integral effect.

In a future work, the developments presented will be used to obtain a simplified modelling of more complex geometries such as a $\mathrm{V}$ dipole, a bow-tie antenna, and a TEM horn.

\section{REFERENCES}

1. L. Yang and G.B. Giannakis, Ultra-wideband communications: An idea whose time has come, IEEE Signal Processing Mag (2004), 26-53.

2. A.M. Attiya and A. Safaai-Jazi, Simulation of ultra-wideband indoor propagation, Microwave Opt Technol Lett 42 (2004), 103-108.

3. T.T. Wu and R.W.P. King, The cylindrical antenna with nonreflecting resistive loading, IEEE Trans Antennas Propagat 13 (1965), 369-373.

4. C.A. Balanis, Antenna theory, $2^{\text {nd }}$ ed., Wiley, New York, 1997.

5. K.P. Esselle and S.S. Stuchly, Pulse-receiving characteristics of resistively loaded dipole antennas, IEEE Trans Antennas Propagat 38 (1990), 1677-1683.

6. S.N. Samaddar and E.L. Mokole, Transient behaviour of radiated and received fields associated with a resistively loaded dipole, Herman et al. (Eds.), Ultra-wideband short electromagnetics, vol. 4, Kluwer Academic-Plenum Publishers, New York, 1999, pp. 165-179.

7. G.S. Smith, Teaching antenna radiation from a time domain perspective, Am J Phys 69 (2001), 829-844.

(C) 2005 Wiley Periodicals, Inc. 